\title{
Multichannel cell detection in microcompartments by means of true parallel measurements using the
} \section{Solartron S-1260}

\author{
T.A. Nguyen ${ }^{1}$, D. Echtermeyer ${ }^{2}$, A. Barthel ${ }^{3}$, G. Urban $^{4}$, and U. Pliquett ${ }^{2,5}$ \\ 1. Department of Physics, Le Quy Don Technical University, Ha Noi, Viet Nam \\ 2. Institut für Bioprozess- und Analysenmeßtechnik, Heilbad Heiligenstadt, Germany \\ 3. Max Planck Institute for Dynamics and Self-Organization, Göttingen, Germany \\ 4. Institut für Mikrosystemtechnik (IMTEK), Albert-Ludwigs-Universität Freiburg, Germany \\ 5. E-mail any correspondence to: uwe.pliquett@iba-heiligenstadt.de
}

\section{Abstract}

Designing proper frontend electronics is critical in the development of highly sophisticated electrode systems. Multielectrode arrays for measuring electrical signals or impedance require multichannel readout systems. Even more challenging is the differential or ratiometric configuration with simultaneous assessment of measurement and reference channels. In this work, an eight-channel frontend was developed for contacting a $2 \times 8$ electrode array $(8$ measurement and 8 reference electrodes) with a large common electrode to the impedance gain-phase analyzer Solartron 1260 (S-1260). Using the three independent and truly parallel monitor channels of the S1260 , impedance of trapped cells and reference material was measured at the same time, thereby considerably increasing the performance of the device. The frontend electronics buffers the generator output and applies a potentiostatic signal to the common electrode of the chip. The applied voltage is monitored using the current monitor of the S-1260 via voltage/current conversion. The frontend monitors the current through the electrodes and converts it to a voltage fed into the voltage monitors of the S-1260. For assessment of the 8 electrode pairs featured by the chip, a relay-based multiplexer was implemented. Extensive characterization and calibration of the frontend were carried out in a frequency range between $100 \mathrm{~Hz}$ and $1 \mathrm{MHz}$. Investigating the influence of the multiplexer and the frontend electronics, direct measurement with and without frontend was compared. Although differences were evident, they have been negligible below one per cent. The significance of measurement using the complex S-1260-frontend-electrode was tested using Kohlrausch's law. The impedance of an electrolytic dilution series
\end{abstract}

was measured and compared to the theoretical values. The coincidence of measured values and theoretical prediction serves as an indicator for electrode sensitivity to cell behavior. Monitoring of cell behavior on the microelectrode surface will be shown as an example.

Keywords: Bioimpedance; current; voltage

\section{Introduction}

The method of measuring electrical impedance is widely used for fast and economic characterization of cell suspensions and tissues. With the increasing sensitivity of instruments and availability of microstructured electrodes, measurements at single cells gain increasing attention [1-5]. Compared to microscopic observation or fluorescence measurement, electrical measurements are label-free, do not need elaborate probe preparation and use comparably simple equipment. Recently, instruments specially designed to analyze single-cell behavior have emerged as an effective approach [6-8]. However, when it comes to high-precision measurement, devices with a high dynamic range of load impedance and frequency range are often considered the better option. In a typical electrical impedance measurement system, the electrical stimulation and simultaneous response monitoring at a pair of electrodes can allow the determination of the impedance. On the other hand, 
tetrapolar configurations use separate electrodes for stimulation and monitoring [9]. Commonly, both voltage and current are monitored using separate input channels. Such devices are well suited for single-channel applications while multichannel configurations are realized with multiplexers.

A special configuration is a differential or ratiometric measurement with an additional reference channel. This can be, for instance, a separate, electrolyte filled measuring chamber with identical dimensions of the chamber used for the measurement but without the test object (e.g. cells).

In principle, it is possible to switch between both chambers and process the absolute values. For simple monitoring of selected behavior of the material under test, hardware solutions for ratiometric or differential preprocessing are favored in most applications.

Especially for scientific questions, absolute impedance values of single cells with respect to electrolyte filled reference electrodes are desirable. Because of the high dynamics of single cells in suspension attaching to electrode surfaces, switching between reference and measuring channel is problematic. For true parallel measurements, two separate monitor channels with identical electrical behavior are required.

The Solartron S-1260 (Schlumberger Ltd, Great Britain, today Ametek Inc.) is a commercial impedance gain-phase analyzer offering a current monitor and two separate voltage monitors [10]. Here we show how to use the features of the S-1260 for simultaneous difference measurement on single-cell level, thereby considerably increasing the capabilities of this device.

\section{Materials and methods}

Electrode chip

Fig. 1 illustrates a packaged electrode chip and the microelectrode arrays (MEAs) in the insert picture. The MEAs are composed of a large common electrode (CE) in the middle surrounded by 32 small electrodes (SE).

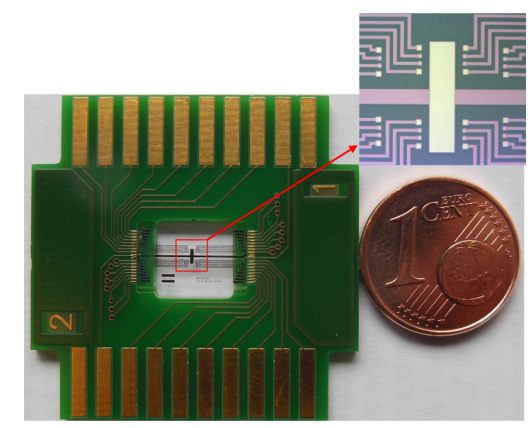

Fig.1: The image of a packaged electrode chip with double side PCB suitable for the multiplexer and the insert image which enlarged microelectrode array.

The CE has a dimension of $350 \mu \mathrm{m} \times 500 \mu \mathrm{m}$ while there are two different sizes of the SE, which are $25 \mu \mathrm{m} \times 25 \mu \mathrm{m}$ and
$50 \mu \mathrm{m} \times 50 \mu \mathrm{m}$, respectively. The SEs are arranged symmetrically on the opposite side of the CE to form pairs of identical structure. In each pair of the SE, one microelectrode is used for measurement of attached cells and the other as reference.

The MEAs are fabricated on a Pyrex substrate using standard microsystem technique [11, 12]. The dimension of one fabricated chip is only $11 \mathrm{~mm} \times 5.3 \mathrm{~mm}$. The sensor is glued to a double side printed circuit board (PCB) which fits with the developed multiplexer.

\section{The fabrication process of the electrode chip}

The fabrication process of the electrode chip was described in detail in our previous works [11, 12]. Briefly, the gold microelectrodes were deposited on the Pyrex wafer using standard microsystem techniques which include: electronbeam evaporator, dry etching, lift-off, plasma-enhanced chemical vapour deposition, photolithography, and dry etching. A thin Chrome layer $(10 \mathrm{~nm})$ was used to enhance the adhesion between the gold layer and the wafer. The passivation layer of $\mathrm{Si}_{3} \mathrm{~N}_{4}$ was deposited over the metal layer.

\section{Impedance measurement}

The S-1260 was used as the main unit. Even after many years on the market, it is one of the most powerful and accurate impedance analyzers. According to the manufacturer, the magnitude resolution is $0.0001 \mathrm{~dB}$ while the phase resolution is $0.01^{\circ}$. The accuracy of the magnitude is given as $0.1 \%$ and the phase accuracy is $0.1^{\circ}$. The internal source generates a sine excitation in frequencies ranging from $30 \mathrm{mHz}$ to $30 \mathrm{MHz}$. The amplitude can be between $10 \mathrm{mV}$ and $3 \mathrm{~V}$ in voltage mode and $10 \mu \mathrm{A}$ up to $100 \mathrm{~mA}$ in current mode. The measurable impedance magnitude ranges from $\mathrm{m} \Omega$ up to $M \Omega$. The detection is based on three independent units - one for current monitoring with a transimpedance input and two identical detectors for two voltage channels which give great freedom for designing frontends. The shields of the inputs can either be connected to ground or actively driven. The stimulus output has in voltage mode an internal resistance of $60 \Omega$.

Since the electrode chip consists of paired electrodes, a true parallel measurement would yield the best reproducibility and precision of the measurement. In principle, the generator (Gen Out) in voltage mode is used for parallel excitation of all channels using the common electrode (Fig.2). The excitation signal is converted into current using a fixed resistor $\left(R_{\text {cur }}\right)$ and fed into the current monitor. It should be noted that the INPUT $I$ is a transimpedance maintaining a virtual ground. The total current through the measuring electrode as well as the reference electrode is converted into voltage with two identically designed transimpedances $\left(\mathrm{OPA} 1 / \mathrm{Z}_{\mathrm{f}, 1}, \mathrm{OPA} 2 / \mathrm{Z}_{\mathrm{f}, 2}\right)$ 
and monitored using both voltage inputs (V1 HI, V2 HI). The operational amplifiers (OPA656, Texas instruments, Dallas, USA) are chosen for high speed and very low signal distortion throughout the output swing. Moreover, due to the JFET input stage, they offer very high input impedance with low parasitic capacitance. The feedback resistors, realized as complex impedances $Z_{f, 1}$ and $Z_{f, 2}$, are plugged for simple adaption to the chip resistance. Owing to the high dynamic range of the impedance magnitude, $Z_{f}$ is not just an ohmic resistor but is a network consisting of two resistors and a capacitor, which mimics in first glace the behavior of the chip. This facilitates nearly the same measurement precision and SNR across the entire frequency range of interest. The inputs and outputs of the Solartron and the frontend are matched to $50 \Omega$, which is the impedance of the RG176-cable used in the device. This ensures independence of the cable length within the desired frequency range.

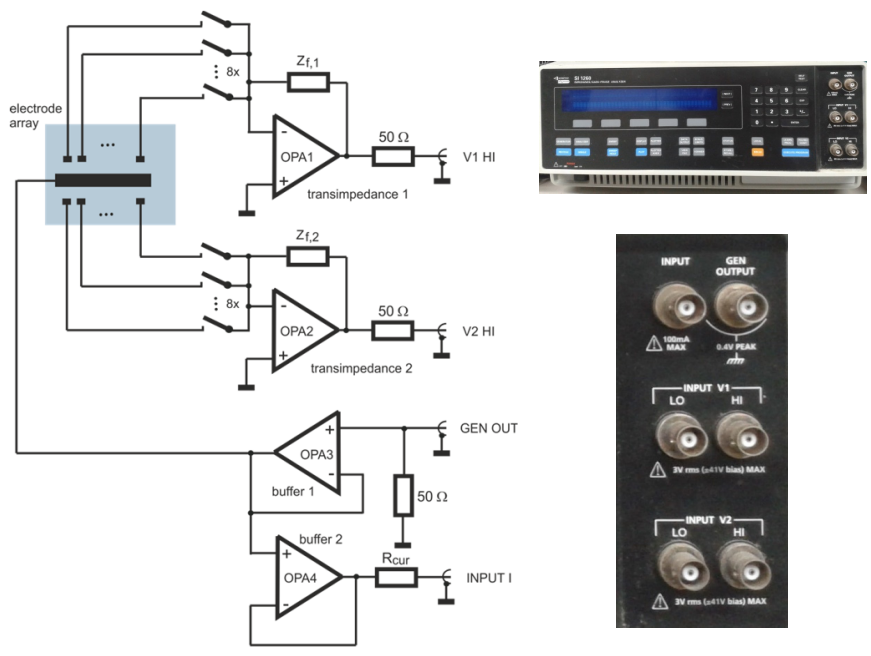

Fig.2: Principle schematic of the true difference frontend (left panel) for Solartron S-1260 (right panel). Each trapping and reference electrode was connected with a relay to the input of the operational amplifiers (OPA1 / OPA2). The shield of the voltage monitors is connected to ground. The measurement channel is connected to the cell trapping electrode.

The inputs are connected as single-ended (V1 HI, V2 HI), thus the V1 LO and V2 LO are left open. The 8 pairs of electrodes were connected to the frontend via a specially designed multiplexer. The major challenges are the high source impedance of the electrodes and the wide measurement bandwidth from $100 \mathrm{~Hz}$ to $1 \mathrm{MHz}$. To optimize the signal quality, we propose the use of mechanical miniature relays instead of solid-state switches (Fig.3).

Owning to their small footprints $(10.6 \mathrm{~mm} \times 7.4 \mathrm{~mm})$, all relays were placed directly on the interface of the chip. The chip is divided into two identical chambers and was bonded onto a PCB adapter with symmetrical contacts at the opposite sides. One side contacted one of both independent chambers of the chip. Thus, by rotating the PCB by 180 degrees, the other side could be assessed.

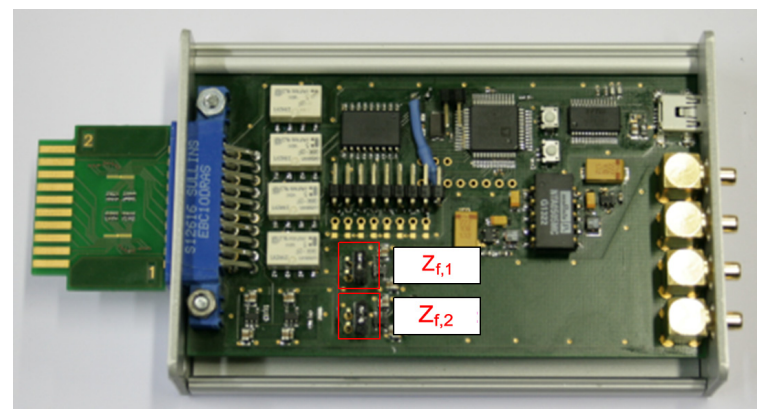

Fig.3: Multiplexer with frontend. At the left side is the receptacle for the adapter PCB with the chip. In the image, a test-chip with fixed resistors is shown. At the right side are the coaxial connectors to the Solartron S-1260 and the USB-connection for the microcontroller. The connectors in the middle of the PCB are for indicator-LED, showing the active electrode pair. A pushswitch is implemented for manually advancing the position of the multiplexer. The feedback resistors are inside the red labeled boxes.

Switching of the relay is controlled by an 8052-based microcontroller (ADuC841, Analog Devices, Natick, MA). For decreasing the thermal noise which is associated with the electrode impedance by

$$
U_{\text {noise }}=\sqrt{4 k T \Delta f Z_{e l}}
$$

a long integration time of $1 \mathrm{~s}$ was set at the instrument. The device is connected to a computer either via GPIB or RS232. A simple routine, written in Matlab (The Mathworks, Natick, $\mathrm{MA})$, controlled the instrument, collected the data and accomplished further processing.

As reference, impedance measurements, subsequently at all working and reference electrodes without the multiplexer, were performed by using the two-electrode configuration of the S-1260. In this case, GEN OUT and V1 HI were bridged together with the counter electrode while the current monitor INPUT I was connected to V1 LO and the electrode under test (cell trapping or reference). The measurement voltage was set to $20 \mathrm{mV}$ and the frequency ranged from $100 \mathrm{~Hz}$ to $1 \mathrm{MHz}$.

\section{Data processing}

The Matlab-routine controls all the instrument settings. After sweeping through the desired frequency range, here from $100 \mathrm{~Hz}$ to $1 \mathrm{MHz}$, all three raw vectors (I, V1, V2) are transferred to the PC.

While the current (INPUT I) is proportional to the voltage applied (GEN OUT) both voltages (V1, V2) refer to the current through the measuring (V1) and the reference electrode (V2) which is converted to a voltage by both transimpedances. 
Because of the output resistance of the Solartron $(60 \Omega)$ and cable matching resistors $(50 \Omega), U_{\text {appl }}$ does not match the voltage set at the instrument, $U_{\text {set }}$, but is $U_{\text {appl }}=0.45 U_{\text {set }}$. The voltage output of a transimpedance as a function of the current, $\mathrm{I}$, is $\mathrm{U}_{\text {out }}=-\mathrm{Z}_{\mathrm{f}} \mathrm{l}$ where $\mathrm{Z}_{\mathrm{f}}$ is the feedback-resistor, which is here a complex network (Fig.3). The values of the feedback network depend on the electrode geometry, the frequency range of interest and the conductivity of the buffer solution. The values used in this study for low conductive electrolytes are:

$$
\mathrm{R}_{\mathrm{s}}=1 \mathrm{k} \Omega, \mathrm{C}_{\mathrm{s}}=470 \mathrm{pF}, \mathrm{R}_{\mathrm{p}}=1 \mathrm{M} \Omega
$$

The values of the elements measured with a precise LCRmeter were used for further calculation.

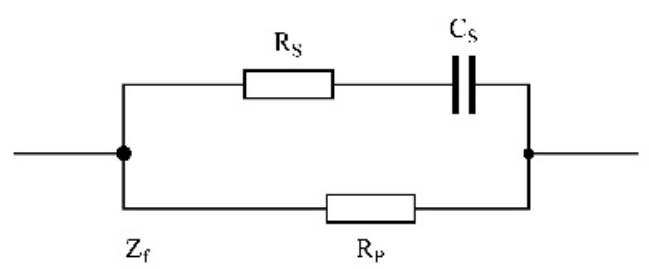

Fig.3: Feedback impedance $Z_{f, 1}=Z_{f, 2}=Z_{f}$ used for the transimpedances in Fig. 2 .

$\mathrm{Z}_{\mathrm{f}}$ can be calculated as

$$
Z_{f}=\frac{R_{p}+j \omega C_{s} R_{s} R_{p}}{1+j \omega C_{s}\left(R_{s}+R_{p}\right)}
$$

where $\omega$ is the angular frequency $\omega=2 \pi f$. Using $Z_{f}$ we find the current through the electrodes as:

$$
I_{\text {meas }}=-\mathrm{V} 1 / Z_{f} \quad \text { and } \quad I_{\text {ref }}=-V 2 / Z_{f}
$$

The impedance between the measurement and the common electrode is

$$
Z_{\text {meas }}=U_{\text {appl }} / I_{\text {meas }}
$$

while the impedance of the reference channel is

$$
Z_{\text {ref }}=U_{\text {appl }} / I_{\text {ref }}
$$

Modeling of the impedance of the cells retrieved from the measurement in order to assess behavior like cell size, permittivity of cytosol, membrane conductivity or capacitance is not the scope of this paper.

\section{Electrolyte dilution series for testing Kohlrausch's law}

For characterization of the electrode chip and the frontend, dilution series of PBS were used. 140 mM PBS (phosphatebuffered saline) was made with $8 \mathrm{~g} \mathrm{NaCl}, 0.2 \mathrm{~g} \mathrm{KCL}, 0.2 \mathrm{~g}$ $\mathrm{KH}_{2} \mathrm{PO}_{4}$, and $1.15 \mathrm{~g} \mathrm{Na}_{2} \mathrm{HPO}_{4}$ in 1 liter of distilled water. $\mathrm{A}$ dilution series of $(4.375,8.75,17.5,35,70$, and 140) $\mathrm{mM}$ PBS was used at room temperature of $22^{\circ} \mathrm{C}$.

As reference and control, the conductivity of the dilution has been measured with a WTW-conductometer with the WTW electrode. All measurements using the electrode chip were done in a frequency range between $100 \mathrm{~Hz}$ and $1 \mathrm{MHz}$ with the multiplexed frontend described here. For comparison, measurements with single electrodes (reference or measurement electrode without frontend) with respect to the common electrode were done. The measurement voltage varied between $10 \mathrm{mV}$ and $200 \mathrm{mV}$ applied at the electrodes.

\section{Ethical approval}

The conducted research is not related to either human or animal use.

\section{Results}

The main purpose of this study is to show the precision and the reliability of the frontend and the multiplexer, but also to test the quality and dynamic range of the impedance chip. Therefore, we compare measurements at known objects like electrolytes with varying dilution between single-channel measurement without any frontend and 8-channel measurements using the device described here. Moreover, the impedance of trapped cells was studied over $5 \mathrm{~h}$ of incubation.

\section{Calibration using dilution series of electrolyte}

The influence of the measurement voltage was negligible between $10 \mathrm{mV}$ and $100 \mathrm{mV}$. All data presented in this paper were done at $20 \mathrm{mV}$ applied across the electrodes. Moreover, for better clarity of the presentation, only data from one pair of electrodes (measuring and reference electrode) are shown. The measurement yielded two traces for each electrode pair. While both could be measured at the same time using the frontend, two measurements were necessary for direct connection to the instrument.

The frequency-dependent impedance shows the typical appearance of mostly resistive impedance (electrolyte) in series with electrode impedance (overwhelmingly capacitive) which is in parallel with a parasitic capacitance (Fig. 4 and 5). The frequency range where the behavior of the electrolyte or objects within this (e.g. cells) can be assessed with maximum precision is where the phase is closest to zero or the slope of the magnitude is minimal. At lower frequency overwhelms the electrode polarization while the object is capacitively shortened by parasitic capacitances at higher frequencies.

As expected, there are essentially no differences between the measurement with and without the frontend, except some higher noise when using a direct connection to the electrode chip (Fig.5). 

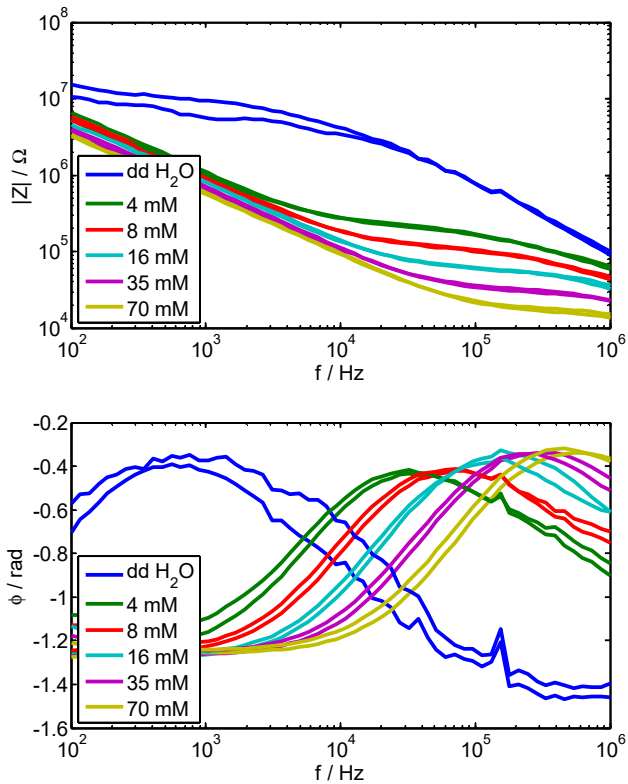

Fig.4: Magnitude and phase angle of a dilution series measured at one electrode pair (measurement and reference electrode) using the multiplexer.
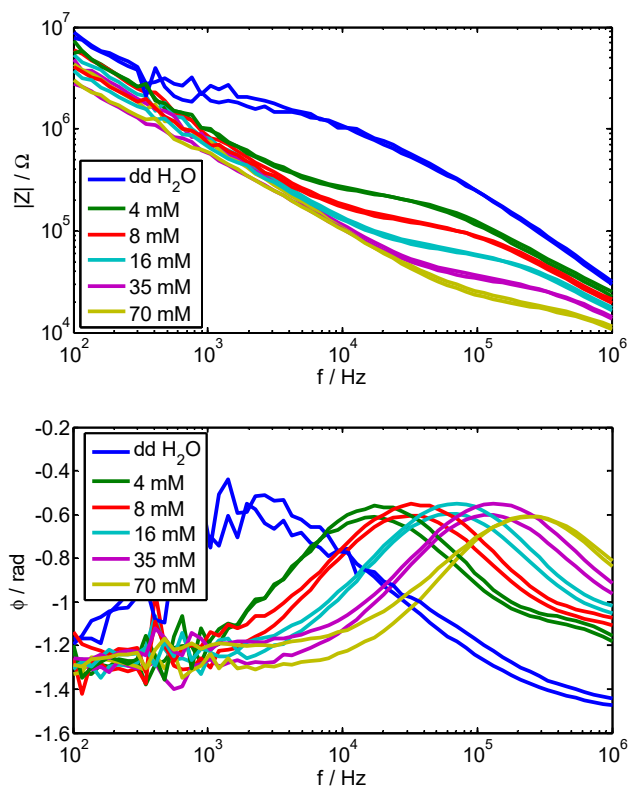

Fig.5: Magnitude and phase angle of a dilution series of PBS (phosphate buffered saline) without multiplexer. $\mathrm{ddH}_{2} \mathrm{O}$ (double distilled water) serves as low conductivity limit. For assessing the measurement and reference electrode, two measurements were necessary.

As seen in Fig. 4 and 5, measurements on electrolyte solution exhibit uncertainties, which are independent of the instrumentation. For a direct comparison, we used a dummy load of $1 \mathrm{M} \Omega$ resistor in parallel with a serial combination of $1 \mathrm{k} \Omega$ and $470 \mathrm{pF}$, which is close to the impedance of the electrode chip filled with $70 \mathrm{mM}$ PBS. Both magnitude and phase show a quite good coincidence (Fig.6).

Although both measurements with and without multiplexer are coincident within 5\%, the multiplexer improves the signal quality, especially in the low-frequency range (Fig.7).
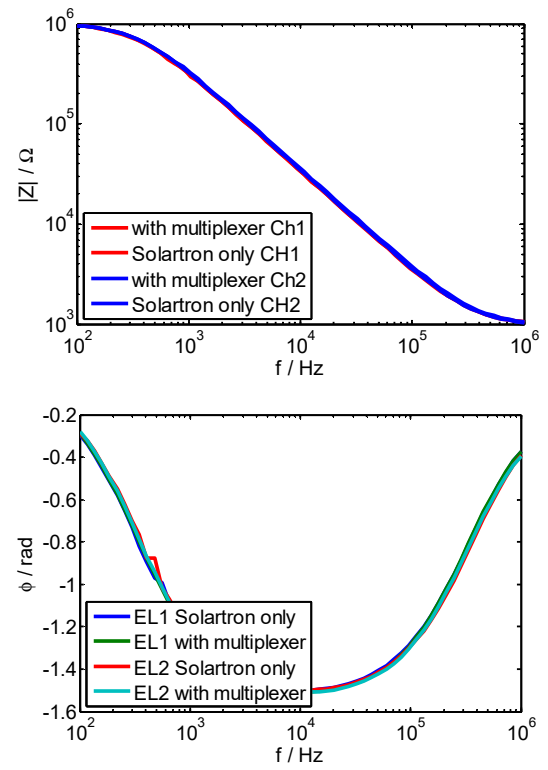

Fig.6: Comparison between measurements with Solartron with and without multiplexer at a dummy load ( $1 \mathrm{M} \Omega$ in parallel with a $470 \mathrm{pF} / 1 \mathrm{k} \Omega$ - serial combination).

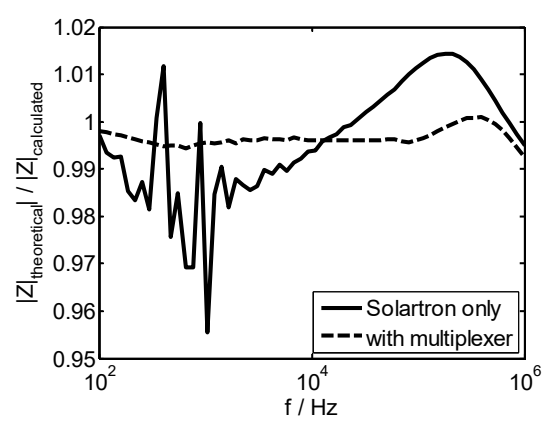

Fig.7: Measured impedance magnitude in relation to the theoretical impedance of a dummy load measured with and without the multiplexer. Obviously, the amplifiers of the frontend, connected with minimum of wiring decrease the noise especially in the low frequency region.

The ultimate test of the quality of an electrode with respect to measurement precision is the test of a natural law like the Kohlrausch's square root law which should be a linear curve when drawing the molar conductivity against the square root of the concentration for strong electrolytes.

$$
\Lambda_{m, 0}=\Lambda_{m}-\alpha \sqrt{c}
$$

The molar conductivity $\Lambda_{\mathrm{m}}$ is the conductivity for the concentration $\Lambda_{m}=\sigma / c$. $\alpha$ is a material constant.

Moreover, when extrapolating to zero concentration, the limiting molar conductivity, $\Lambda_{m, 0}$, found in tables for a given temperature and electrolyte, should be hit.

This is quite well fulfilled for the measurement using a reference system, here a WTW-conductometer (LF-3000, WTW, Weilheim, Germany) (Fig.8, circles). Because the conductivity of the electrolyte influences the frequency window where it can be measured with the highest precision, we accessed the conductivity at the frequency, 
where the phase angle is closest to zero. This yields data shown in Fig. 6 as asterix. It is obvious that the slope of the curve differs slightly, which is attributed to the difference by measuring at a fixed frequency (WTW, $400 \mathrm{~Hz}$ ) using electrodes especially made for conductivity measurements and various frequencies using a microelectrode.

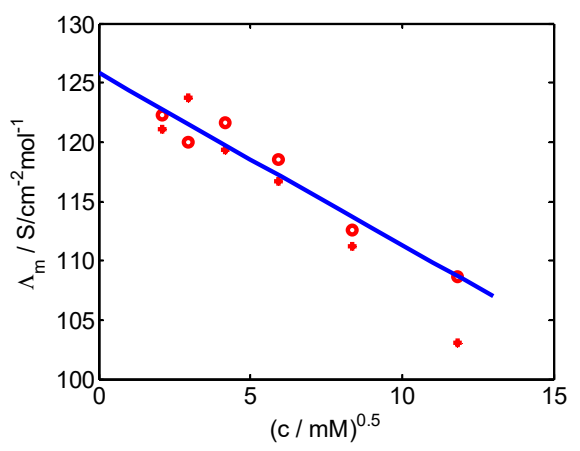

Fig.8: Test of the dilution series of PBS (phosphate buffered saline) using a WTW-conductometer with WTW-electrode (o) and from impedance measurement at the frequency with phase closest to zero $\left({ }^{*}\right)$. The theoretical $\Lambda_{\mathrm{m}}$ (blue line) for PBS has the limit at concentration $\mathrm{CPBS}_{\mathrm{PB}}=0 \mathrm{M}$ of $126 \mathrm{~S} / \mathrm{cm}^{-2} \mathrm{~mol}^{-1}$.

\section{Change of impedance of trapped cells during incubation}

After the fabrication and bonding process, the chip surface was first cleaned by oxygen plasma and then adhered with polydimethylsiloxane (PDMS) to form the microfluidic chip. A flow of fibronectin was injected into the microchannel and incubated for 1.5 hours at room temperature. To trap a single cell on the microelectrode surfaces, a flow of cells (breast cancer cell, MDA-MB-231) suspended with a concentration of $10^{6}$ cells $/ \mathrm{ml}$ was injected into the microchannel. The details of the experimental procedure can be found in our previous works [11, 12].

The impedance measurement was performed to monitor the cell behaviors such as cell attachment and cell adhesion. Fig. 9 illustrates the normalized difference of (a) the impedance magnitude and (b) the phase between working electrodes with trapped cells and the reference ones. The red line represents the measured values taken immediately after the single cells being trapped while the blue line represents the measured values taken 5 hours after incubation. The error bars show the standard deviation of 7 electrodes. The $8^{\text {th }}$ electrode did not trap a cell and was omitted.

The absolute values of impedance are available but not very informative when looking at changes at cell level. Therefore, the data are normalized to the impedance of the electrodes in contact with the suspension medium, which is PBS in this case.

Here, we present the difference of the normalized magnitude and the phase difference. The magnitude difference $\Delta|\mathrm{Z}|$ was calculated as

$$
\Delta|Z|=\frac{\left|Z_{\text {cell,t }}\right|}{\left|Z_{\text {cell,PBS }}\right|}-\frac{\left|Z_{\text {ref }, 0}\right|}{\left|Z_{\text {ref,PBS }}\right|}
$$

while the phase difference is

$$
\Delta \varphi=\left(\varphi_{c e l l, t}-\varphi_{c e l l, P B S}\right)-\left(\varphi_{r e f, 0}-\varphi_{r e f, P B S}\right)
$$

The indices cell and ref refer to the cell trapping and the reference electrode. PBS stays for the PBS-filled chamber, 0 for the time immediately after cell trapping and $t$ for the incubation time.
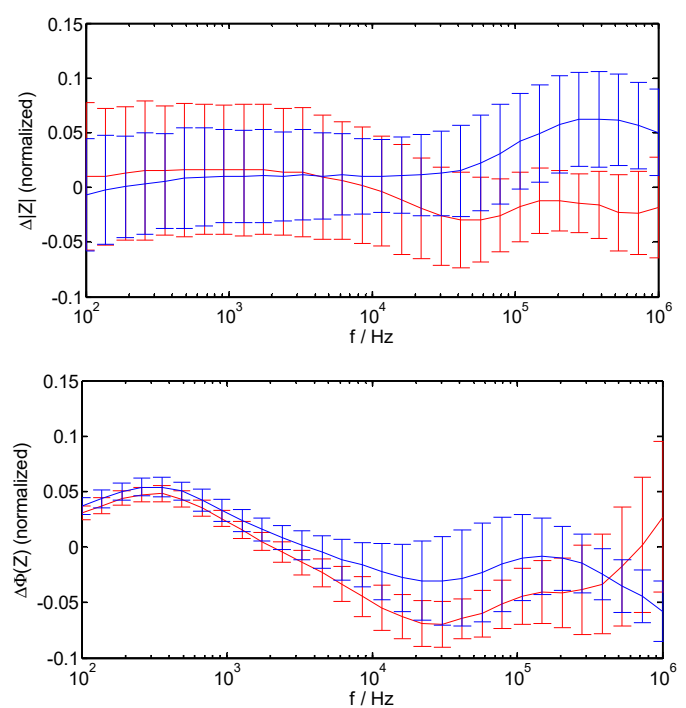

Fig.9: Normalized difference of (a) the impedance magnitude and (b) the phase angle difference between working electrodes with trapped cells and the reference ones. The red line indicates the measurement immediately after trapping the cells and the blue line was obtained $5 \mathrm{~h}$ after incubation. The error bars show the standard deviation of 7 electrodes. The 7th out of the 8 electrodes did not trap a cell. It is therefore not shown and omitted in the calculation.
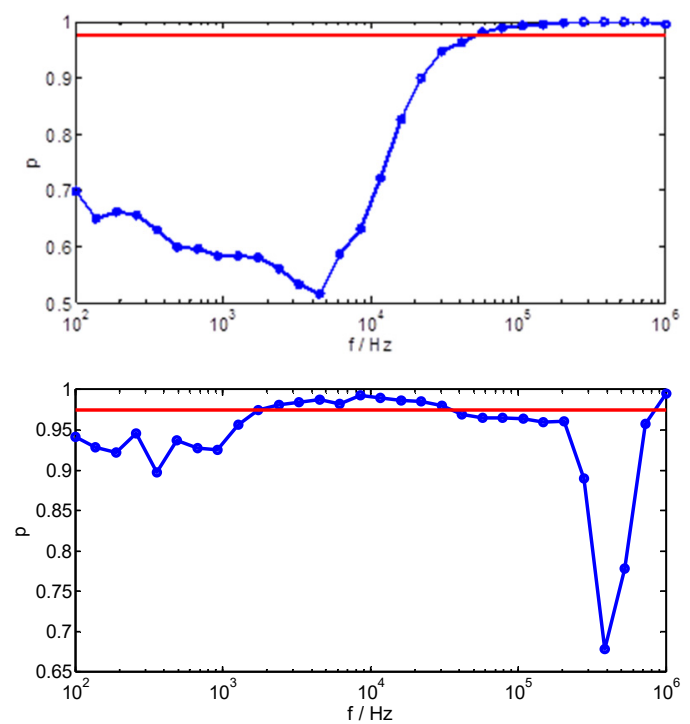

Fig.10: $\alpha$-Quantiles for double sided t-test (A) magnitude of impedance and $(B)$ phase. The red line indicated the significance level of $5 \%(0.975)$. The mean value is statistically different for frequencies with $p$-value above this line. 
With exception of electrode 7, all electrodes trapped a cell. Because there have not been any changes, results for electrode 7 are omitted. For better clarity, results from all electrodes are averaged and shown together with the standard deviation (Fig.9).

\section{Discussion}

The use of a multiplexer together with a matched frontend for microelectrode arrays is a useful add-on for a device like the impedance gain-phase analyzer Solartron S-1260. In principle, it would be enough to measure either the voltage across the object when applying a current or vice versa. This, however, implies that the applied stimulus is completely determined, i.e. the voltage or current is stable over the frequency range and the phase angle is always zero (Fig.11). Real measurements of the stimulus, however, show that this is not the case, requiring the monitoring of the stimulus as well.

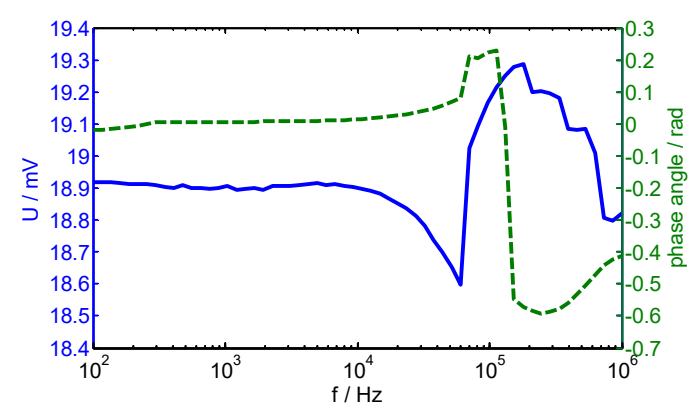

Fig.11: Magnitude (solid line) and phase angle (dashed line) of the applied voltage.

If the frontend exhibits different group delays within different channels (e.g. I, V1, V2), compensation for this electronics-based uncertainty is required. As visible from Fig.8, the surge in phase around $100 \mathrm{kHz}$, which is evidently caused by an intrinsic feature of the Solartron, not by the frontend, illustrates the necessity of proper compensation. This compensation should be applied for all other channels. Since the impedance is the ratio of voltage and current, it is fully compensated in impedance measurements.

The use of a cell constant for the description of the electrode behavior is nonsense because the frequency window where the behavior of the material between the electrodes can be assessed is small and depends on the conductivity. Therefore, the conductivity at the phase close to zero was used for determining the sensitivity of the electrode system (cell constant), which is the slope of the $\sigma(|Y|)$ - function where $s$ is the electrolyte conductivity and $|\mathrm{Y}|$ is the magnitude of the measured conductance. For the electrode chip, a cell constant of $1.24 \cdot 10^{6} \mathrm{~cm}^{-1}$ was found. Although the optimum lies around $0.67 \mathrm{~cm}^{-1}$, this value is acceptable for a microelectrode.

Due to the extremely short wiring and the excellent behavior of the operational amplifiers used, parasitic capacitances could be minimized, thereby extending the useful frequency range of the measurement chain (Fig.12).
While the magnitude of the impedance falls dramatically towards higher frequency when the S-1260 was directly attached to the chip, the plateau corresponding to the specific resistance of the electrolyte is extended when using the frontend. Moreover, the uncertainties in the low frequency range are considerably reduced. Although not ideal, which would be a horizontal line throughout the frequency range used here, the use of the frontend improves the overall performance considerably.

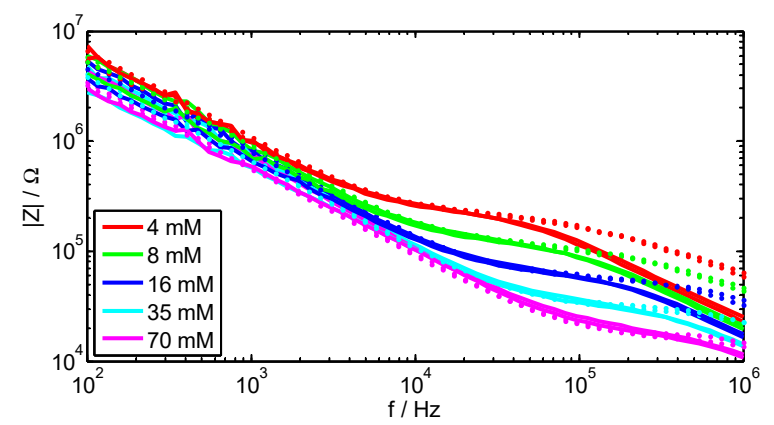

Fig.12: Magnitude of the impedance measured with multiplexed frontend (dots) and by direct attachment of the chip to the S-1260.

\section{Conclusion}

In this work, an eight-channel frontend as add-on for the Solartron S-1260 was successfully developed for simultaneous measurement of eight pair of electrodes. A series of experiments were performed with PBS to compare the measurements with Solartron S-1260 along with and without multiplexer. Although both measurements are coincident within $5 \%$, the multiplexer improves the signal quality to better than $1 \%$ with respect to theoretical values, especially in the low-frequency range. The experiment for monitoring single-cell behavior was also carried out to evaluate the efficiency of the frontend. The impedance signals compare well to our previous works when measuring with only one pair of electrodes using Solartron S-1260 but measurement time for all eight channels degrease by an order of magnitude while the noise decreased considerably.

\section{Acknowledgement}

This research is funded by Vietnam National Foundation for Science and Technology Development (NAFOSTED) under grant number 103.99-2017.65.

Ethical approval

The conducted research is not related to either human or animal use.

\section{Conflict of interest}

Authors state no conflict of interest.

\section{References}

1. Gimsa J, Muller T, Schnelle T, Fuhr G. Dielectric spectroscopy of single human erythrocytes at physiological ionic strength: dispersion of the cytoplasm. Biophys J. 1996;71(1):495-506. https://doi.org/10.1016/S0006-3495(96)79251-2 
2. Gawad S, Schild L, Renaud PH. Micromachined impedance spectroscopy flow cytometer for cell analysis and particle sizing. Lab Chip. 2001;1(1):76-82. https://doi.org/10.1039/b103933b

3. Holmes D, Pettgrew D, Reccius CH, Gwyer JD, van Berkel C, Holloway J, et al. Leukocyte analysis and differentiation using high speed microfluidic single cell impedance spectroscopy. Lab on a Chip. 2009;9:2881-9. https://doi.org/10.1039/b910053a

4. Sun T, van Berkel C, Green NG, Morgan H. Digital signal processing methods for impedance microfluidic cytometry. Microfluidics and Nanofluidics. 2009;6(2):179-87. https://doi.org/10.1007/s10404-008-0315-3

5. Thein M, Asphahani F, Cheng A, Buckmaster R, Zhang M, Xu J. Response characteristics of single-cell impedance sensors employed with surface-modified microelectrodes. Biosensors and Bioelectronics. 2010;25:1963-9. https://doi.org/10.1016/j.bios.2010.01.023

6. Hassan U, Bashir R. Electrical cell counting process characterization in a microfluidic impedance cytometer. Biomedical Microdevices. 2014 2014/10/01;16(5):697-704. https://doi.org/10.1007/s10544-014-9874-0

7. Mernier G, Duqi E, Renaud P. Characterization of a novel impedance cytometer design and its integration with lateral focusing by dielectrophoresis. Lab on a Chip. 2012;12(21):4344-9. https://doi.org/10.1039/c2lc40551b

8. Haandbæk N, Bürgel SC, Heer F, Hierlemann A. Characterization of subcellular morphology of single yeast cells using high frequency microfluidic impedance cytometer. Lab on a Chip. 2014;14(2):369-77. https://doi.org/10.1039/C3LC50866H

9. Grimnes S, Martinsen OG. Bioimpedance and Bioelectricity Basics: Academic Press; 2015. https://doi.org/10.1016/B978-0-12-411470-8.00011-8

10. AMETEK.Inc. 2020; Available from: https://www.ameteksi.com/products/materials-testingsystems/1260a-impedance-gain-phase-analyzer.

11. Nguyen TA, Yin T-I, Reyes D, Urban GA. Microfluidic chip with integrated electrical cell-impedance sensing for monitoring single cancer cell migration in three-dimensional matrixes. Analytical Chemistry. 2013;85(22):11068-76. https://doi.org/10.1021/ac402761s

12. Anh-Nguyen $T$, Tiberius $B$, Pliquett $U$, Urban GA. An impedance biosensor for monitoring cancer cell attachment, spreading and drug-induced apoptosis. Sensors and Actuators A: Physical. 2016;241:231-7. https://doi.org/10.1016/j.sna.2016.02.035 